\section{OP0249 MULTI-BIOMARKER DISEASE ACTIVITY AND AUTOANTIBODY STATUS LEAD TO COST EFFECTIVE TAPERING ALGORITHMS IN RHEUMATOID ARTHRITIS PATIENTS IN SUSTAINED REMISSION}

M. Hagen ${ }^{1}$, M. Englbrecht ${ }^{1}$, J. Haschka ${ }^{2}$, M. Reiser ${ }^{1}$, A. Kleyer ${ }^{1}$, A. Hueber ${ }^{1}$, B. Manger $^{1}$, C. Figuereido ${ }^{3}$, J. Fogagnolo Cobra ${ }^{3}$, H.-P. Tony ${ }^{4}$, S. Finzel ${ }^{5}$, S. Kleinert ${ }^{6}$, J. Wendler ${ }^{6}$, F. Schuch ${ }^{6}$, M. Ronneberger ${ }^{6}$, M. Feuchtenberger $^{7}$, M. Fleck ${ }^{8}$, K. Manger ${ }^{9}$, W. Ochs ${ }^{10}$, M. Schmitt-Haendle ${ }^{10}$, H.-M. Lorenz ${ }^{11}$, H. Nuesslein ${ }^{12}$, R. Alten ${ }^{13}$, J. Henes ${ }^{14}$, K. Krueger ${ }^{15}$, G. Schett ${ }^{1}$, J. Rech ${ }^{1}$ ${ }^{1}$ University of Erlangen-Nuremberg, Erlangen, Germany; ${ }^{2}$ St. Vincent Hospital, Vinforce Study Group, Medical University of Vienna, Vienna, Austria; ${ }^{3}$ Institutio de Rheumatologia, Sao Paolo, Brazil; ${ }^{4}$ University of Wuerzburg, Internal Medicine 2, Wuerzburg; ${ }^{5}$ University Medical Center Freiburg, Rheumatology and Clinical Immunology, Freiburg; ${ }^{6}$ Rheumatology Clinical Practice Erlangen, Erlangen; ${ }^{7}$ Rheumatology Practice and Department of Internal medicine 2, Clinic Burghausen, Burghausen; ${ }^{8}$ Asklepios Medical Center, Department of Rheumatology and clinical Immunology, Bad Abbach; ${ }^{9}$ Rheumatology Practice Bamberg, Bamberg; ${ }^{10}$ Rheumatology Practice Bayreuth, Bayreuth; ${ }^{11}$ University of Heidelberg, Medicine 5, Heidelberg; ${ }^{12}$ Rheumatology Practice Nuremberg, Nuremberg; ${ }^{13}$ Schlosspark Klinik, Internal Medicine/Rheumatology, Berlin ${ }^{14}$ University of Tuebingen, Centre for Interdisciplinary Clinical Immunolog, Tuebingen; ${ }^{15}$ Praxiszentrum St.Bonifatius, Munich, Germany

Background: Achieving remission is the ultimate treatment goal in patients with rheumatoid arthritis (RA). With the development and wider use of highly effective disease modifying anti-rheumatic drugs (DMARD) about half of RA patients reach the disease remission state (1), raising the question about tapering or stopping anti-rheumatic treatment and appropriate predictors (2).

Objectives: To analyse the effect of a risk-stratified DMARD tapering algorithm based on multiple-biomarker disease activity (MBDA) score and anti-citrullinated protein (ACPA) status for successful DMARD tapering and treatment cost reduction in RA patients in sustained remission enrolled in the prospective randomized controlled RETRO study $(3,4)$.

Methods: MBDA scores and ACPA status were determined in the baseline samples of 146 patients in sustained remission. Patients either continued DMARDs (arm1), tapered dose by $50 \%$ (arm 2) or stopped DMARDs after tapering (arm 3) for one year according to the RETRO study protocol. Direct treatment costs (including testing costs at baseline) were evaluated every three months. MBDA and ACPA status were used as predictors creating a risk-stratified tapering algorithm based on relapse rates.

Results: RA patients with a low MBDA score $(<30)$ and negative ACPA showed lowest relapse risk (19\%). With either single positivity for ACPA or moderate/high MBDA scores $(\geq 30)$ relapse risk increased and was high in double-positive patients $(61 \%)$. In MBDA negative $(<30)$ and MBDA single-positive $(>30)$ groups, DMARD tapering appears feasible. Considering only patients that did not flare, costs for synthetic and biologic DMARDs in the MBDA-negative and singlepositive groups $(n=41)$ would have been $123.751,29 €$ for full-dose treatment over one year. Tapering and stopping DMARDs in this low-risk relapse groups allowed a reduction of $92.821,50 €(-75 \%)$ of DMARD costs. Average reduction of DMARD costs per patient were $2.350,08 €$ in the double negative (MBDA/ACPA-) and single negative (MBDA- /ACPA+) group and 1.761,43€ in the MBDA single positive (MBDA+ /ACPA-) group.

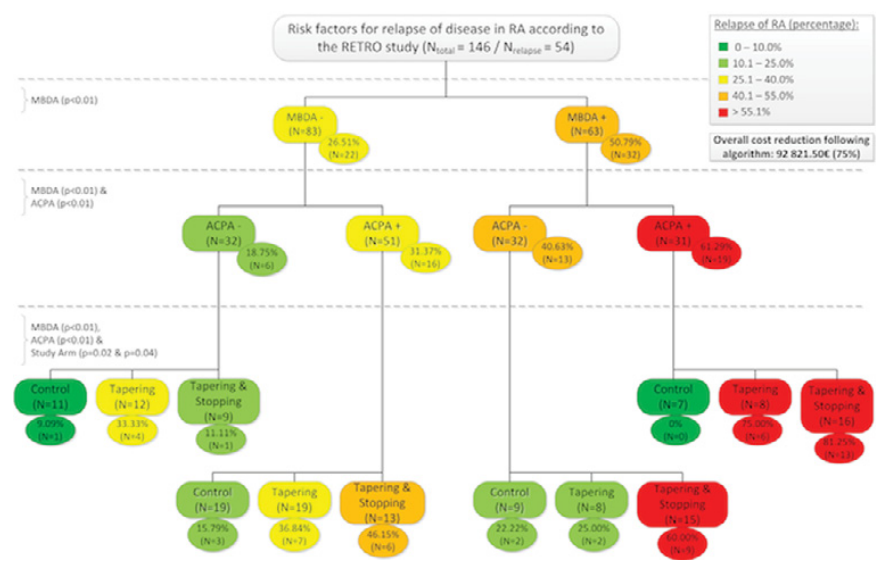

Conclusions: Combining MBDA score and ACPA status allows risk stratification for successful DMARD tapering and cost-effective use of biologic DMARD. Given that previous data of the RETRO have shown that patients relapsing after tapering their DMARDs respond well to their reintroduction, a stratified tapering and stopping of DMARDs is not only a cost economic but also clinically feasible strategy.

References:

[1] Aga AB et al. Ann Rheum Dis. 2013;74:381-8.

[2] Schett G et al. Ann Rheum Dis. 2016 Aug;75(8):1428-373.

[3] Haschka J et al, Ann Rheum Dis. 2016;75:45-51.
[4] Rech J et al, Ann Rheum Dis. 2015; Oct. 19

Disclosure of Interest: None declared

DOI: 10.1136/annrheumdis-2017-eular.2988

\section{OP0250 CAN RANKL SERUM LEVELS PREDICT FUTURE PROGRESSION TO RHEUMATOID ARTHRITIS IN ACPA NEGATIVE PATIENTS?}

A. Burska ${ }^{1}$, J. El-Jawhari ${ }^{1}$, A.L. Tan ${ }^{1}$, R. Wakefield ${ }^{1}$, H. Marzo-Ortega ${ }^{2}$, P. Conaghan ${ }^{1}$, P. Emery ${ }^{1}$, F. Ponchel ${ }^{1}$, J. Freeston ${ }^{1,2,3}$. ${ }^{1}$ LIRMM , University of Leeds; ${ }^{2}$ NIHR Leeds Musculoskeletal Biomedical Research Centre;

${ }^{3}$ Rheumatology, Leeds Teaching Hospitals NHS Trust, Leeds, United Kingdom

Background: Making the earliest diagnosis of rheumatoid arthritis (RA) is crucial to initiate treatment and prevent further disease progression and joint damage. Despite recent advances with the discovery and integration of anti-cyclic citrullinated protein antibody (ACPA) in classification criteria, there is still an unmet need for new diagnostic biomarkers, notably for ACPA-negative disease. Power Doppler ultrasound has been shown to identify poor prognosis disease in ACPA negative patients.

Objectives: The receptor-activator-nuclear-factor- $\kappa B$ axis (RANK/RANKL) is known to regulate bone homeostasis. The aim of this pilot study is to establish whether serum RANKL levels in people with early inflammatory arthritis are associated with RA diagnosis at follow-up and to evaluate the added value of RANKL with ultrasound for early RA diagnosis.

Methods: Serum from 298 subjects (95/204 Male/Female) was collected at the baseline participant visit to the Leeds Early Arthritis clinic. Demographic (age, gender symptom duration) and clinical data (swollen and tender joint counts (SJC, TJC), CRP, DAS28, rheumatoid factor (RF) and ACPA, shared epitope (SE)) were collected.

A commercial ELISA (BioVENDOR) was used to measure RANKL. Ultrasound of 26 joints (bilateral elbows, wrists, MCP 2-3, PIP 2-3, knees, ankles and MTP 1-5) was performed at baseline recording summative scores for power Doppler (PD), grey scale hypertrophy (GS) and erosions (ERO).

Results: At 1 year follow-up, 151 patients had a confirmed diagnosis of RA (EULAR 2010 criteria) and 147 were classified as non-RA (undifferentiated arthritis, other inflammatory diagnoses or non-persistent inflammation). All routinely used biomarkers were associated with RA diagnosis (ACPA, RF, SE, TJC, SJC, CRP, DAS28, $p<0.0001$ ), as were imaging biomarkers (PD, GS, ERO, $p<0.001$ ). RANKL levels were significantly higher in RA (RA 1002.4 $\pm 1053.2 \mathrm{pmol} / \mathrm{L}$, non-RA $339.2 \pm 451.5 \mathrm{pmol} / \mathrm{L}, \mathrm{p}<0.0001)$. A regression analysis suggested that four parameters were sufficient to account for all associations with RA: RANKL, age, SJC, and PD with $75.3 \%$ accurate prediction. An AUROC analysis suggested a cut-off for each parameter and a score was calculated, adding 1 point for each of the factors (RANKL $>700$, age $>62, T P D>3, S J C>4)$. This score predicted RA with an AUROC of $0.782((0.23-0.840), p<0.0001)$. The same analysis repeated for ACPA negative patients only $(n=193)$ showed similar results, providing accurate diagnosis of RA (77.6\% correct by regression) and with an AUROC of 0.774 ((0.690-0.858), $\mathrm{p}<0.0001)$.

Conclusions: A score incorporating RANKL, age, SJC and PD showed good predictive value for non-RA when low and for RA when high. Furthermore, the analysis redone in ACPA-negative patients performed particularly well for predicting RA with a good AUROC value.

Disclosure of Interest: None declared

DOI: 10.1136/annrheumdis-2017-eular.3226

\section{OP0251 INCIDENCE OF KNEE AND HIP REPLACEMENTS IN RHEUMATOID ARTHRITIS PATIENTS FOLLOWING INTRODUCTION OF BIOLOGICAL DMARDS: AN INTERRUPTED TIME SERIES ANALYSIS USING NATIONWIDE HEALTH CARE REGISTERS}

R. Cordtz ${ }^{1,2}$, S. Hawley ${ }^{3}$, D. Prieto-Alhambra ${ }^{3}$, L.E. Kristensen ${ }^{2}$,

S. Overgaard ${ }^{4}, A$. Odgaard ${ }^{5}$, L. Dreyer ${ }^{1,2}{ }^{1}$ Center for Rheumatology and Spine Diseases, Gentofte, Rigshospitalet; ${ }^{2}$ The Parker Institute, Bispebjerg and Frederiksberg Hospital, Copenhagen, Denmark; ${ }^{3}$ Musculoskeletal Pharmacoand Device Epidemiology, NDORMS, University of Oxford, Oxford, United Kingdom; ${ }^{4}$ Department of Orthopaedic Surgery and Traumatology, Odense University Hospital, Odense; ${ }^{5}$ Depepartment of Orthopaedics, Herlev and Gentofte Hospital, Copenhagen, Denmark

Background: Previous data have been conflicting regarding a possible impact of treatment with biological DMARDs (bDMARDs) on the need for total knee replacement $(T K R)$ and total hip replacement $(T H R)$ in patients with rheumatoid arthritis (RA) ${ }^{1}$

Objectives: To investigate impact of national guidelines recommending bDMARD treatment for RA on the secular trends of TKR and THR among incident RA patients compared with matched general population controls (GPC) in Denmark. Methods: Nationwide register-based interrupted time-series analysis using the National Patient Register and Civil Registration System.

$R A$ : incident patients diagnosed at a rheumatology department from 1996-2011. GPC: 10 individuals matched to each RA patient on age, sex and municipality. Outcome: First TKR and THR, respectively. 